\title{
The trend of prevalence of pain in Korea from 2005 to 2016
}

\author{
Sang-Hyeon Cho ${ }^{1}$, Yong-Min Kim², Jae-Ho Lee ${ }^{1}$, Hyun-Soo Kim¹, and Jae-Seok Song ${ }^{3}$ \\ 'Department of Anesthesiology and Pain Medicine, Gangneung Asan Hospital, Gangneung, Korea \\ ${ }^{2}$ Department of Chemical and Biological Engineering, College of Engineering, Seoul National University, Seoul, Korea \\ ${ }^{3}$ Department of Preventive Medicine, Catholic Kwandong University College of Medicine, Gangneung, Korea
}

Received February 12, 2020

Revised June 25, 2020

Accepted July 6, 2020

Handling Editor: Yeon Dong Kim

\section{Correspondence}

Jae-Seok Song

Department of Preventive Medicine,

Catholic Kwandong University College

of Medicine, 24 Beomil-ro 579beon-gil,

Gangneung 25601, Korea

Tel: +82-33-649-7469

Fax: +82-33-641-1074

E-mail: songjs@cku.ac.kr
Background: Korean society is afflicted with rapid aging. Aging is a risk factor for pain, and pain can reduce patients' quality of life. Thus, adequate management and monitoring of changing trends accompanying the demographic shift are highly valuable. However, this study was conducted because no studies have investigated the recent changes in the prevalence of pain.

Methods: The extent of the prevalence of pain was determined by questions related to quality of life based on the data derived from the Korea National Health and Nutrition Survey (KNHNS) from 2005 to 2016. The annual frequencies of the pain group and severe pain group were calculated using the survey questionnaire. Multiple logistic regression analysis was performed to determine possible differences in prevalence by year.

Results: The prevalence of pain in all populations was 30.6\% in 2005 and $18.9 \%$ in 2016. The average prevalence from 2005 to 2016 was $21.9 \%$. A declining trend occurred over time with an odds ratio of 0.929 per year (95\% Cl: 0.921-0.938). The prevalence of severe pain was $2.35 \%$ in 2005 and $1.88 \%$ in 2016 . Likewise, a decrease was observed over time, with an odds ratio of 0.920 per year at $95 \% \mathrm{Cl}$ 0.901-0.939. The decline in age-/sex-stratified analysis also showed a statistically significant trend in all groups.

Conclusions: The prevalence of pain in Korean society, based on the KNHNS, has declined since 2005. Such a trend was observed in all ages and sexs, and was most significant in the elderly.

Key Words: Aging; Data Analysis; Health Surveys; Nutrition Surveys; Pain; Population Dynamics; Quality of Life; Risk Factors.

\section{INTRODUCTION}

Korean society has been rapidly aging since 2000 , when the proportion of the elderly population passed $7 \%$. Recently in 2017 , the elderly population exceeded $14 \%$ and Korean society has been classified as an aged society [1]. Various forecasts suggest that the aging of the society will further accelerate, and that Korea will enter the era of the super-aged society. In response to such demographic changes, it is imperative to predict and respond to various health problems that may occur in the elderly population.

Older adults are more likely to have pain problems. However, it is also true that pain is poorly managed in older adults in general [2], which can lead to a deterioration in their quality of life. Moreover, untreated pain issues can limit daily activities, including economic and recreational activities, which can gradually lead to a vicious cycle $[3,4]$. In that sense, understanding the incidence and trends in (a) This is an open-access article distributed under the terms of the Creative Commons Attribution Non-Commercial License (http://creativecommons.org/licenses/by-nc/4.0/), which permits unrestricted non-commercial use, distribution, and reproduction in any medium, provided the original work is properly cited.

(c) The Korean Pain Society, 2020
Author contributions: Sang-Hyeon Cho: Writing/manuscript preparation; Yong-Min Kim: Writing/manuscript preparation; Jae-Ho Lee: Writing/ manuscript preparation; Hyun-Soo Kim: Writing/manuscript preparation; Jae-Seok Song: Supervision. 
the prevalence of pain in Korea will be the starting point in managing the quality of life of the increasing elderly population.

The importance of pain management has recently been widely accepted, and a large amount of medical expenses is being used to manage pain and develop advanced methods of pain management $[5,6]$. Countries such as the United Kingdom, the United States of America (USA), and Australia have included items related to pain in their national surveys to provide analytical results [7]. In Korea, data necessary for the analysis of pain prevalence are based on the Survey of Living Conditions and Welfare Needs of Korean Older Persons, Korea National Health and Nutrition Survey (KNHNS), and the Korean Longitudinal Study of Aging [3]. However, despite the rapid aging observed in the Korean society, few reports analyzing recent trends in pain prevalence are currently available. This study analyzed the change in prevalence of pain in Korea, based on the KNHNS data generated annually at a national level for all age groups.

\section{MATERIALS AND METHODS}

\section{Data}

This study is a retrospective, longitudinal, populationbased, observational survey, based on data obtained from 2005 to 2016. The KNHNS is conducted by the Korea Centers for Disease Control and Prevention (KCDC). The 2006 data were excluded, since the survey was not conducted. The KNHNS is a nationwide health and nutrition survey conducted annually in accordance with Article 16 of the National Health Promotion Act. The sampling frame of the survey is based on the most recent population housing census data available at the time of sampling. In order to use the latest information that reflects the current characteristics of the population, the public housing price data were added to supplement the basic extraction framework and improve the population inclusion rate. It ensured adequate representation of the target population in the sample [8]. The survey was held based on face-to-face interviews in mobile checkup centers.

The sample sizes used in years $2005 / 2007-2009 / 2010$ 2012/2013-2015/2016 were 33,805/24,871/25,534/22,948/ 8,150 , respectively, accounting for 115,308 in total. Participants aged below 19 years were excluded from this study, and 87,908 samples were analyzed. During the process of data collection, the data of 23,118 samples were missed due to non-response or refusal of the subjects. Finally, a total of 64,790 samples were used for statistical analysis. Demographic data of the samples in each year are listed in
Table 1. This study was approved by the Institutional Review Board of the Gangneng Asan Hopsital, University of Ulsan (GNAH 2020-04-033).

\section{Definition of variables}

The study used Euro quality of life-5 dimension (EQ-5D) of the KNHNS, which reflects the quality of life. EQ-5D quantifies participants' generic health status based on self-completion of survey items related to five dimensions (mobility, self-care, usual activities, pain/discomfort, and anxiety/depression). Among the five dimensions, this study referred to the item related to pain/discomfort. The item was designed to categorize the participants' status of pain/discomfort according to three levels: "I have no pain or discomfort", "I have moderate pain or discomfort", and "I have extreme pain or discomfort". Participants who reported moderate pain/discomfort or extreme pain/ discomfort were defined as the pain group, and those who complained of extreme pain/discomfort were further classified into a severe pain group. The prevalence was divided into subgroups according to population, sex, and age. The age groups were classified as follows: 1) age between 19 and 44 years, 2) age between 45 and 64, and 3) age more than 65 years.

\section{Statistical analysis}

Weightings offered by the KCDC were applied to the data to enhance the representativeness of the sample to the Korean population. The annual frequencies of the pain group and severe pain group were calculated using the survey questionnaire. The aforementioned weightings were applied to each datum to deduct the weighted frequency using SURVEYFREQ (SAS Institute, Cary, NC, USA), and the

Table 1. Demographic Sample Data Used in This Study

\begin{tabular}{|c|c|c|c|c|c|c|c|}
\hline \multirow[b]{2}{*}{ Year } & \multicolumn{3}{|c|}{ Male } & \multicolumn{3}{|c|}{ Female } & \multirow[b]{2}{*}{ Total } \\
\hline & $19-44$ & $45-64$ & $\begin{array}{c}\text { Age } 65 \\
\text { and } \\
\text { over }\end{array}$ & $19-44$ & $45-64$ & $\begin{array}{c}\text { Age } 65 \\
\text { and } \\
\text { over }\end{array}$ & \\
\hline 2005 & 1,088 & 918 & 366 & 1,569 & 1,082 & 542 & 5,565 \\
\hline 2007 & 518 & 443 & 307 & 749 & 595 & 434 & 3,046 \\
\hline 2008 & 1,266 & 1,006 & 585 & 1,747 & 1,296 & 940 & 6,840 \\
\hline 2009 & 1,412 & 1,152 & 698 & 1,816 & 1,481 & 979 & 7,538 \\
\hline 2010 & 1,139 & 976 & 630 & 1,528 & 1,290 & 789 & 6,352 \\
\hline 2011 & 1,009 & 991 & 651 & 1,382 & 1,299 & 892 & 6,224 \\
\hline 2012 & 905 & 928 & 673 & 1,321 & 1,246 & 922 & 5,995 \\
\hline 2013 & 1,037 & 890 & 558 & 1,333 & 1,203 & 771 & 5,792 \\
\hline 2014 & 897 & 832 & 656 & 1,237 & 1,184 & 871 & 5,677 \\
\hline 2015 & 886 & 926 & 645 & 1,084 & 1,252 & 839 & 5,632 \\
\hline 2016 & 1,020 & 932 & 695 & 1,327 & 1,272 & 883 & 6,129 \\
\hline
\end{tabular}


prevalence of each group was calculated and expressed as a percentile. The analysis was conducted separately by age or sex. Errors related to differences in average age by the year were corrected. Multiple logistic regression analysis was performed to determine possible differences in prevalence by year. Odds ratio was calculated for each model including age and sex. All data were analyzed. Data were interpreted as significant at a $P$ value less than 0.05 using SAS ver. 9.4 (SAS Institute).

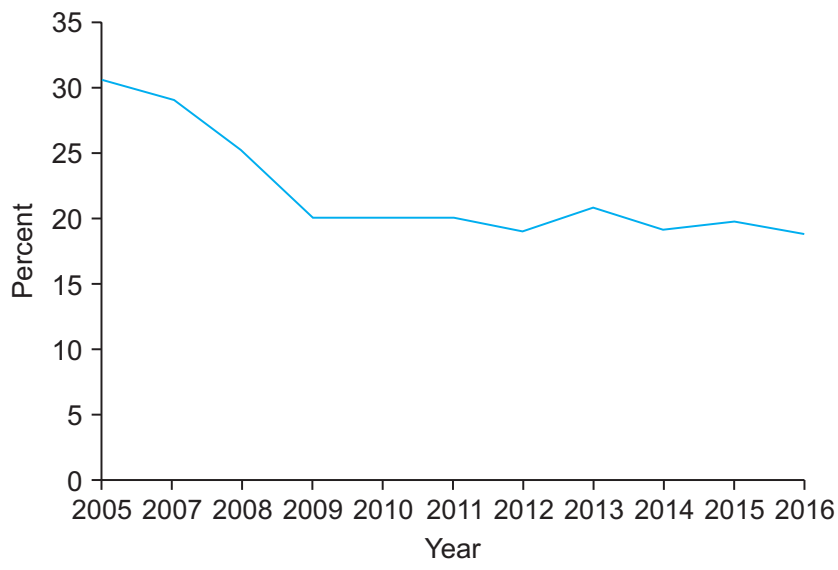

Fig. 1. The trend in prevalence of pain.

Table 2. The Odds Ratio for Pain and Severe Pain according to Variables

\begin{tabular}{lcc}
\hline Variable & $\begin{array}{c}\text { Odds ratio in pain } \\
(95 \% \mathrm{Cl})\end{array}$ & $\begin{array}{c}\text { Odds ratio in severe pain } \\
(95 \% \mathrm{Cl})\end{array}$ \\
\hline Sex & $1.837(1.750-1.930)$ & $2.164(1.905-2.459)$ \\
Age & $1.035(1.033-1.037)$ & $1.075(1.071-1.080)$ \\
Year & $0.929(0.921-0.938)$ & $0.920(0.901-0.939)$ \\
\hline
\end{tabular}

Cl: confidence interval.

\section{RESULTS}

The prevalence of pain ranged from $30.6 \%$ in 2005 to $18.9 \%$ in 2016. The average prevalence from 2005 to 2016 turned out to be $21.9 \%$ (Fig. 1). The prevalence of pain showed a decreasing trend with an odds ratio of 0.929 , and a $95 \%$ confidence interval (CI) of 0.921-0.938, indicating statistical significance (Table 2). The prevalence of severe pain was $2.35 \%$ in 2005 and $1.88 \%$ in 2016 (Fig. 2). The prevalence of severe pain also decreased with an odds ratio of 0.920 , and was statistically significant with a $95 \%$ CI of 0.901-0.939 (Table 2).

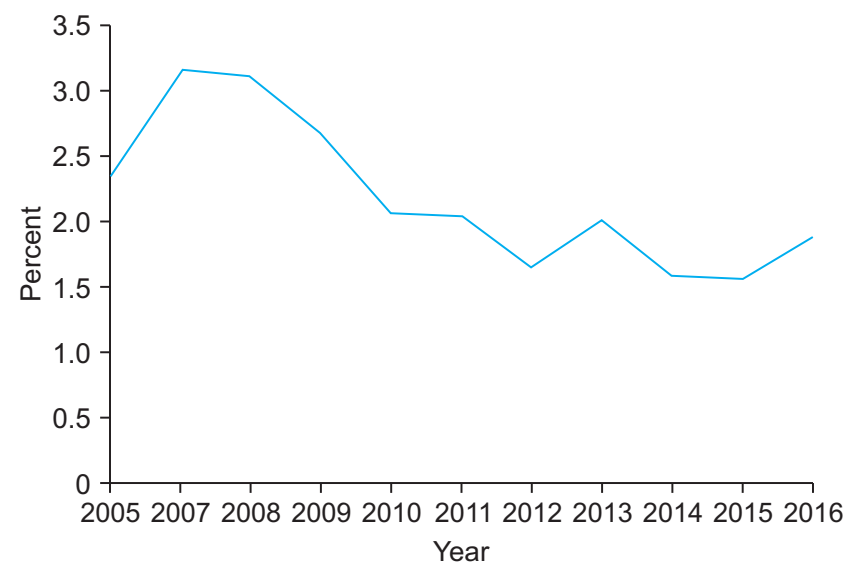

Fig. 2. The trend in prevalence of severe pain.

Table 3. The Average Prevalence of Pain according to Sex and Age Groups from 2005 to 2016

\begin{tabular}{lcccccccc}
\hline & \multicolumn{3}{c}{ Male } & & \multicolumn{3}{c}{ Female } \\
\cline { 2 - 4 } \cline { 6 - 7 } Severity & $19-44$ & $45-64$ & $\begin{array}{c}\text { Age 65 } \\
\text { and over }\end{array}$ & $19-44$ & $45-64$ & $\begin{array}{c}\text { Age 65 } \\
\text { and over }\end{array}$ \\
\hline Pain (\%) & 11.7 & 18.5 & 30.5 & & 17.4 & 30.2 & 50.1 \\
Severe pain (\%) & 0.38 & 1.42 & 4.25 & & 0.56 & 2.36 & 12.08 \\
\hline
\end{tabular}

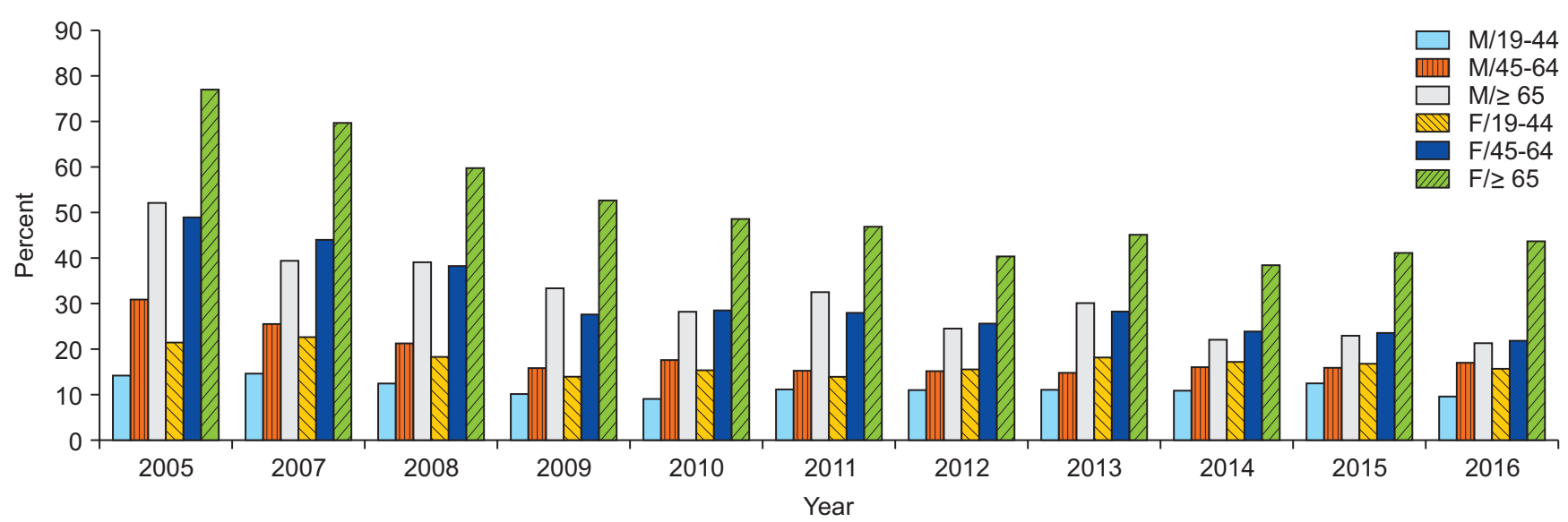

Fig. 3. The trend in prevalence of pain by sex and age groups: 19-44 years old, $45-64$ years old, and 65 years old or older. 
The average prevalence of pain in the subgroups according to age and sex is shown in Table 3. The annual change in the prevalence of pain in each group is shown in Fig. 3 . The number of subjects suffering from pain in the female group was 1.837-fold higher than in the male group (95\% CI, 1.750-1.930). The prevalence increased by 1.035 -fold per year as age increased, which was statistically significant (95\% CI, 1.033-1.037) (Table 2). Furthermore, the odds ratio per year in all groups was less than 1 , which suggested that the prevalence of pain decreased over time, with a statistical significance. The results also indicate that the decreased prevalence was more significant in the groups at higher ages (Table 4).

For severe pain, the prevalence changed in each group as shown in Fig. 4. The female group complained of 2.164fold higher pain compared with the male group (95\% CI, 1.905-2.459). The prevalence increased by 1.075 -fold each year older, which was statistically significant (95\% CI, 1.071-1.080) (Table 2). Furthermore, there was a decreasing trend in the prevalence of severe pain among all groups except in the male group under 45 years of age (Table 4 ).

\section{DISCUSSION}

Pain is a crucial factor determining quality of life. Pain is generally divided into acute and chronic types, based on a 3-month period [9]. This classification is based on whether or not pain alleviation occurs within the normal time of healing, and the mechanism and direction of treatment also differ between the two types of pain $[9,10]$. Many studies focus only on the prevalence of chronic pain. However, regardless of the duration of pain, poorly relieved pain is an essential cause of functional impairment and deterioration of quality of life in any age group [2]. Especially in the elderly, management of not only chronic pain, but also acute pain, is seriously difficult [2]. Therefore, this study focused on analyzing a broader incidence and trend of pain including both acute and chronic pain in diverse groups according to age or sex.

The main objective of this study was to analyze the recent trends in the prevalence of pain in Korea, which is considered an increasingly aging society. A reflection of changes in population structure was a crucial element in the analysis. The focus was on sample representativeness in each subgroup. The KNHNS uses well-sampled big data, by incorporating the most recent population housing census data as the basic extraction framework, and a stratified cluster sample method, to define each sample size in accordance with the proportion of each subgroup [11]. Thus, the sample representativeness is significantly improved compared to random sampling. The KNHNS provided a 


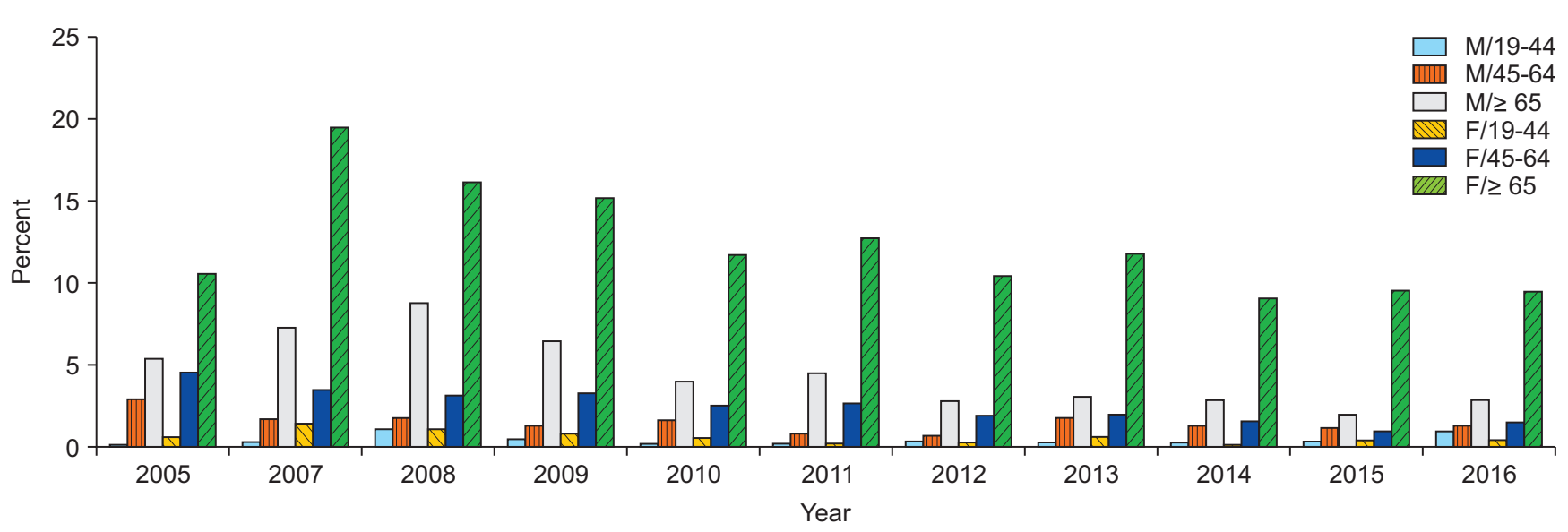

Fig. 4. The trend in prevalence of severe pain by sex and age groups: 19-44 years old, 45-64 years old, and 65 years old or older.

large database, which was entirely necessary for our analysis.

Pain is highly subjective, and the same degree of pain is perceived as higher or lower depending on the individual. Moreover, the degree of pain is assessed differently, depending on the methods and questionnaires adopted by different survey organizations [12]. Thus, many investigations of the prevalence of pain show differences depending on the survey methods used. In this regard, this study was based on a single survey over a long period. Although the results of assessment may have limitations in objectively reflecting the degree of pain experienced by the population, the survey retained the assessment criteria for the 10 -year period, which generated adequate data for exploration of the national trends in the prevalence of pain. The decreasing trends of pain prevalence in Korea over the past ten years are thus considered clinically significant.

According to the results of this study, the prevalence of pain in the entire Korean population over age 19 was $21.9 \%$ on average, from 2005 to 2016. According to the study investigated by the Korea Health Panel, the prevalence of pain among 58,151 adults over the age of 18 years from 2009 to 2013 was $29.8 \%$ [5]. In the case of studies investigating chronic pain in other countries, the prevalence of chronic pain among 6,000 individuals in the total population was $39.3 \%$ in Japan in 2011 [13]. In Hong Kong, the prevalence of chronic pain in 2007 was $35.2 \%$ in a sample size of 5,001 in the general population [14]. The prevalence of "pain" in the Canadian population ranged from $15.1 \%$ to $18.9 \%$ during the years 1994-2008, according to big data research using stratified samples [15]. In the USA, according to a random sampling survey, $20.4 \%$ of the total population reported experiencing chronic pain [6]. However, the differences in the definition of pain in each study should be considered when interpreting the results.

According to the analysis in this study, the prevalence of pain was $30.6 \%$ in 2005 and $18.9 \%$ in 2016 , showing a de- creasing trend with an odds ratio of 0.929 . The prevalence of severe pain averaged $2.17 \%$ from 2005 to 2016 , decreasing from $2.35 \%$ in 2005 to $1.88 \%$ in 2016 , with an odds ratio of 0.920 . The prevalence of pain in all age-/sex-subgroups showed a significant decreasing trend over the period. Especially in the female group 65 years old or older, the decreasing trend in the prevalence of pain was the greatest (odds ratio, 0.881) and the prevalence decreased significantly between age groups as the age increased (Table 4). According to the Korea National Statistical Office, the population 65 years old or above was 4.32 million in 2005 (8.9\% of total population), and 6.76 million in 2016 (13.2\% of total population) [16]. Although the proportion of the aged population increased, the prevalence of pain actually showed a decreasing trend. In the case of Canada, the prevalence of pain did not show any statistically significant trend during 1994-2008 [15].

It is widely known that the elderly and female population are exposed to a higher degree of pain [7]. The results of this study are parallel with such observations: the prevalence increased by 1.035 times per year as the age increased and the female group experienced 1.837 times more pain than the male group as shown in Table 2. Therefore, the conclusion of this study is, aging was a factor increasing the prevalence of pain and although the elderly population increased, the prevalence of pain decreased through all age subgroups, especially in the elderly population (Figs. 1, 3).

Although it is difficult to define the causes of such a decreasing trend in this study, diverse factors may have affected the results. Some of the causes might be the increase in medical service availability and medical expenses. In fact, the frequency of medical use in patients with pain was 5.858-fold higher [17], and the medical expenses of patients with pain showed a relatively high increase [5].

Against the background of such phenomena, the changes in pain perception by patients and the development of 
diverse and state-of-the-art interventions, are available. Recent advances in access to health information, including those associated with control of pain, are broadcast via diverse media compared to the past [18]. Besides, according to researchers, patients report that they are ready to adopt such information spontaneously, and implement it into actual practice [19]. Moreover, there have been remarkable developments in the diagnosis and treatment of pain, which have been applied to different types of pain, resulting in favorable outcomes for many pain patients [20]. These factors appear to have played a role in such phenomena.

This study was based on the KNHNS. This annual, wellstandardized and controlled national survey has the advantage of high reliability. Another advantage is the high representativeness of the sample since it was extracted from the latest population housing census data, which reflects recent demographic changes. Moreover, the statistically significant decrease shown in such large-scale data surveys strongly suggests the persistence of such phenomena.

As mentioned above, the present study adopted "stratified cluster sampling" rather than "random sampling" to improve the representativeness of the subgroups of subjects distinguished by sex and age. However, the approach required knowledge of population, higher costs, and time for the sampling [11]. In addition, the data from a longterm period were necessary to identify the trends in the prevalence of pain. Therefore, big data was employed for the analysis. However, the present study had a few limitations, attributable to the inability of researchers to design the questions contained in the questionnaire.

Our study has several limitations. First, a more specific analysis according to the lasting period of pain was not conducted. The focus was thus directed towards general pain, and not chronic pain, which has recently attracted substantial attention. If it was considered important to determine whether or not the pain lasted more than three months, our results would have overestimated than the prevalence of chronic pain. Second, the analyses on the origins of the pain and subgroups according to the type of underlying disease were impossible. Further research based on data with more subgroup information will enable us to specifically detect the causes of the decreasing trend of prevalence of pain. Besides, the researches could also provide us with the information on whether pain of different types are under control and being treated appropriately, or require further attention due to inadequate care.

In conclusion, according to our study, the prevalence of pain was $30.6 \%$ in 2005 and $18.9 \%$ in 2016 , showing a statistically significant decreasing trend. The decreasing trend appeared in all age and sex subgroups, and was most significant in the elderly population.

\section{CONFLICT OF INTEREST}

No potential conflict of interest relevant to this article was reported.

\section{FUNDING}

No funding to declare.

\section{ORCID}

Sang-Hyeon Cho, https://orcid.org/0000-0001-9363-2573

Yong-Min Kim, https://orcid.org/0000-0001-6866-4135

Jae-Ho Lee, https://orcid.org/0000-0002-2965-5794

Hyun-Soo Kim, https://orcid.org/0000-0001-6817-7214

Jae-Seok Song, https://orcid.org/0000-0001-5583-182X

\section{REFERENCES}

1. Jang IY, Lee HY, Lee E. Geriatrics Fact Sheet in Korea 2018 from national statistics. Ann Geriatr Med Res 2019; 23: 50-3.

2. Schofield P. Pain in older adults: epidemiology, impact and barriers to management. Rev Pain 2007; 1: 12-4.

3. Jung-Choi K, Park J, Kim N, Park H. Status of chronic pain prevalence in the Korean adults. Public Health Weekly Report 2015; 8: 728-34.

4. Kawai K, Kawai AT, Wollan P, Yawn BP. Adverse impacts of chronic pain on health-related quality of life, work productivity, depression and anxiety in a community-based study. Fam Pract 2017; 34: 656-61.

5. Shin SM. Prevalence and trends of pain associated with chronic diseases and personal out-of-pocket medical expenditures in Korea. Korean J Pain 2017; 30: 142-50.

6. Dahlhamer J, Lucas J, Zelaya C, Nahin R, Mackey S, DeBar $\mathrm{L}$, et al. Prevalence of chronic pain and high-impact chronic pain among adults - United States, 2016. MMWR Morb Mortal Wkly Rep 2018; 67: 1001-6.

7. Fayaz A, Croft P, Langford RM, Donaldson LJ, Jones GT. Prevalence of chronic pain in the UK: a systematic review and meta-analysis of population studies. BMJ Open 2016; 6: e010364.

8. Kweon S, Kim Y, Jang MJ, Kim Y, Kim K, Choi S, et al. Data resource profile: the Korea National Health and Nutrition Examination Survey (KNHANES). Int J Epidemiol 2014; 43: 69-77. 
9. Treede RD, Rief W, Barke A, Aziz Q, Bennett MI, Benoliel R, et al. A classification of chronic pain for ICD-11. Pain 2015; 156: 1003-7.

10. Grichnik KP, Ferrante FM. The difference between acute and chronic pain. Mt Sinai J Med 1991; 58: 217-20.

11. Sharma G. Pros and cons of different sampling techniques. Int J Appl Res 2017; 3: 749-52.

12. Hawker GA, Mian S, Kendzerska T, French M. Measures of adult pain: Visual Analog Scale for Pain (VAS Pain), Numeric Rating Scale for Pain (NRS Pain), McGill Pain Questionnaire (MPQ), Short-Form McGill Pain Questionnaire (SF-MPQ), Chronic Pain Grade Scale (CPGS), Short Form-36 Bodily Pain Scale (SF-36 BPS), and Measure of Intermittent and Constant Osteoarthritis Pain (ICOAP). Arthritis Care Res (Hoboken) 2011; 63 Suppl 11: S240-52.

13. Inoue S, Kobayashi F, Nishihara M, Arai YC, Ikemoto T, Kawai T, et al. Chronic pain in the Japanese community-prevalence, characteristics and impact on quality of life. PLoS One 2015; 10: e0129262.

14. Wong WS, Fielding R. Prevalence and characteristics of chronic pain in the general population of Hong Kong. J Pain 2011; 12: 236-45.
15. Reitsma ML, Tranmer JE, Buchanan DM, Vandenkerkhof EG. The prevalence of chronic pain and pain-related interference in the Canadian population from 1994 to 2008. Chronic Dis Inj Can 2011; 31: 157-64.

16. Statistics Korea. Population projections for Korea [Internet]. Daejeon: Statistics Korea; 2019. Available at: http://kosis.kr/ statHtml/statHtml.do?orgId=101\&tblId=DT_1BPA002\&vw_ cd=\&list_id=\&scrId=\&seqNo=\&lang_mode=ko\&obj_var_ id=\&itm_id=\&conn_path=E1.

17. Jeong EK, Kwak YH, Song JS. Influences of chronic pain on the use of medical services in South Korea. J Korea Contents Assoc 2015; 15: 363-9.

18. Kim C. Analysis of broadcasting review trends after enacting the Guidelines on promotional mass media appearances by physicians. J Korean Med Assoc 2016; 59: 763-70.

19. Lee HJ, Park JY. Correlations between old people's watching of television programs and practice of health behaviors. J Korea Contents Assoc 2011; 11: 294-301.

20. Kim YD, Moon HS. Review of medical dispute cases in the pain management in Korea: a medical malpractice liability insurance database study. Korean J Pain 2015; 28: 254-64. 